\title{
Preparation, characterization and systemic application of self-assembled hydroxyethyl starch nanoparticles-loaded flavonoid Morin for hyperuricemia therapy
}

This article was published in the following Dove Press journal:

International Journal of Nanomedicine

\author{
Jianbo $\mathrm{Li}^{\prime}$ \\ Yang Yang' \\ Likang Lu \\ Qiujin $\mathrm{Ma}^{2}$ \\ Jinjie Zhang ${ }^{2}$ \\ 'Institute of Medical and \\ Pharmaceutical Sciences, Zhengzhou \\ University, Zhengzhou, People's \\ Republic of China; ${ }^{2}$ School of \\ Pharmaceutical Sciences, Zhengzhou \\ University, Zhengzhou, People's \\ Republic of China
}

Correspondence: Jinjie Zhang School of Pharmaceutical Sciences, Zhengzhou University, No 100 Kexue Road, Zhengzhou, Henan Province 45000I, People's Republic of China Tel +8637I 6778 I908 Email liger1029@।26.com
Background: Morin, one of the most widely distributed flavonoids in plants, has been identified as a potent antihyperuricemic agent. Its poor water solubility and fast in vivo clearance, however, have limited its application in the treatment of hyperuricemia. In this study, a novel amphiphilic polymer (hydroxyethyl starch-deoxycholic acid [HES-DOCA]) was synthesized to overcome these limitations.

Methods: HES-DOCA conjugates with various substitution degrees were prepared by chemical grafting DOCA to HES through ester formation. The structures of the conjugates were confirmed by infrared spectroscopy and ${ }^{1} \mathrm{H}-\mathrm{NMR}$. Physicochemical characterizations of HES-DOCA nanoparticles-loaded Morin (Morin/HES-DOCA-NPs) were studied using dynamic light scattering and transmission electron microscopy (TEM). In vitro release studies were performed to evaluate the release properties of Morin from the NPs. Subsequently, in vivo pharmacokinetic parameters of Morin/HES-DOCA-NPs were investigated in Wistar rats through intravenous administration ( $2 \mathrm{mg} / \mathrm{kg}$, equivalent to Morin). Antihyperuricemic efficacy of the NPs was evaluated in a rat hyperuricemic model.

Results: The optimized HES-based amphiphilic polymer contained approximately 10 DOCA groups per 100 anhydroglucose units of HES, which can spontaneously self-assemble to form spherical NPs as demonstrated by TEM images. Morin/HES-DOCA-NPs were monodispersed (polydispersity index $=0.05$ ) with a mean diameter of $197 \mathrm{~nm}$ and exhibited a zeta potential of $-14 \mathrm{mV}$. The use of DOCA as the polymer's hydrophobic segment enabled high drug loading efficiency (15.6\%). After systemic administration, Morin/HES-DOCA-NPs exhibited significantly longer half-life and higher systemic exposure (elimination half-life and area under the plasma concentration-time curve) compared with free drug Morin. In a rat hyperuricemic model, treatment with Morin/HES-DOCA-NPs demonstrated superior therapeutic efficacy over Morin in decreasing serum uric acid level, increasing the uricosuric action, as well as attenuating hyperuricemia-associated inflammation in kidney of rats.

Conclusion: Collectively, these findings suggest that the novel HES-based NP formulation of Morin may have great potential for clinical treatment of hyperuricemia.

Keywords: HES-DOCA, drug loading efficiency, antihyperuricemic activities, half-life

\section{Introduction}

Hyperuricemia, marked by high serum levels of uric acid, is the major etiological cause of gout and the most common inflammatory arthritis in human. ${ }^{1,2}$ Modulation of the overproduction and/or underexcretion of uric acid represents a rational approach 
in the management of hyperuricemia. ${ }^{3}$ Morin $\left(3,5,7,2^{\prime}, 4^{\prime}\right.$ pentahydroxyflavone), one of the flavonoids that is extensively present in most medicinal herbs, is well known for its anti-inflammatory, anticancer and antioxidant activities. ${ }^{4-6}$ Previous studies have shown that Morin remarkably inhibited uric acid production and reduced renal urate absorption in vitro, demonstrating its potent antihyperuricemic efficacy. ${ }^{7,8}$ However, translation of the in vitro findings into effective in vivo antihyperuricemic activities remains lacking. In particular, available data on antihyperuricemic activities of Morin through intravenous administration are presently scarce.

The practical use of Morin is limited mainly due to its poor water solubility and short serum half-life $(0.37 \mathrm{~h})$, as determined in our present study. This may result in insufficient drug levels in plasma to produce a clinically relevant effect. ${ }^{9}$ Our group previously developed a self-nanoemulsifying drug delivery system-loaded Morin phospholipid complex with enhanced water solubility and oral absorption. ${ }^{9,10}$ However, the nanoemulsions failed to extend the serum half-life of Morin, due to extensive first-pass metabolism of Morin after oral administration. Recently, Morin hydrate niosomes have been successfully prepared with improved water solubility and drug entrapment efficiency but also showed minimal effect on the half-life of Morin after intravenous injection. ${ }^{11}$ Moreover, the drug loading (DL) efficiency was very low $(<4 \%)$, causing excessive uses of excipients.

Recognizing these issues associated with the in vivo delivery of Morin, we hypothesized that a systemically administered Morin formulation with both prolonged circulation half-life and high DL capacity would overcome its poor water solubility and best facilitate its use in hyperuricemia treatment. Hydroxyethyl starch (HES), a semi synthetic derivative of starch polysaccharide, is widely used as a colloidal plasma volume expander due to the negligible toxicity and immunity to the body. ${ }^{12,13}$ Compared with native starch, the abundant groups of HES is expected to have longer body circulation time and is more feasible for modification or functionalization. ${ }^{14}$ Although HES is considered as a naturally derived substitute for the often used water-soluble PEG, a few HES-based nanoplatforms have been reported. Previously, HES has been hydrophobically modified with stearic acid. ${ }^{12}$ However, HES-stearic acid conjugates show poorly self-assembled properties and wide size distribution, which is undesirable for drug delivery. Therefore, the strategy to fabricate stable HES-based nanoparticles (NPs) for efficient drug delivery remains an alluring but great challenge.
We here report a novel HES-based amphiphilic polymer loaded with flavonoid Morin for in vivo treatment of hyperuricemia in a rat model. Deoxycholic acid (DOCA), a natural compound with well-known amphiphilicity to form micelles in water, was selected as the hydrophobic segment of the polymer. It was expected that the introduction of DOCA into HES would induce better self-assembled performance of NPs than stearic acid. Our results demonstrated that the systemically delivered HES-based nanoformulation significantly improved DL, prolonged in vivo circulation time of Morin and thus enhanced therapeutic efficacy for treating hyperuricemia.

\section{Materials and methods \\ Materials}

HES (130/0.4) was purchased from Nanjing Duly Biotech Co., Ltd. N,N-dicyclohexylcarbodiimide (DCC), Nhydroxysuccinimide (NHS) and DOCA were purchased from Aladdin Reagent Inc. (Shanghai, People's Republic of China). Pyrene was purchased from Sigma-Aldrich. The other reagents were of analytical grade and used without further purification.

Male Wistar rats were provided by the Laboratory Animal Center of Zhengzhou University (Zhengzhou, People's Republic of China). All animal experiments were approved by the Institutional Animal Care and Ethic Committee of Zhengzhou University, according to the criteria outlined in the "Guide for the Care and Use of Laboratory Animals Chinese Version" (2006). The rats were allowed to acclimate for at least 1 week and fasted overnight with free access to water prior to experiments.

\section{Synthesis of HES-DOCA conjugates}

Amphiphilic HES-DOCA conjugates were prepared by chemical grafting DOCA to HES through ester formation. Briefly, the carboxyl groups of DOCA were reactivated by DCC (1.5 eq.) and NHS (1.5 eq.) in anhydrous tetrahydrofuran at room temperature for $24 \mathrm{~h}$. The formed precipitate (dicyclohexylurea) was removed by filtration. Excess amount of n-hexane was added to the light yellow filtrate under constant stirring. After filtration, the residue was washed with n-hexane and dried under vacuum overnight to obtain NHS ester of DOCA (DOCA-NHSE). In total, $250 \mathrm{mg}$ of HES $(0.6 \mathrm{mmoL})$ was dried at $80^{\circ} \mathrm{C}$ under vacuum overnight before dissolving in $10 \mathrm{~mL}$ of redistilled N,N-dimethylformamide in a round bottomed flask. To the solution were added different amounts of DOCA-NHSE and N,N-diisopropylethylamine, 
in which the molar ratio of DOCA-NHSE to HES varied from 0.2 to 2 . The flask was tightly sealed and stirred for $24 \mathrm{~h}$ at $40^{\circ} \mathrm{C}$. Then, the reactant mixture was dialyzed against excess amount of distilled water/ethanol (1:2, v/v) for 3 days and distilled water for 1 day using a dialysis bag (molecular cutoff $20 \mathrm{kDa}$ ), respectively. After being freeze-dried, HES-DOCA conjugate was obtained as a white powder.

\section{Characterization of HES-DOCA conjugates}

The chemical structure of HES-DOCA conjugates was confirmed using ${ }^{1} \mathrm{H}-\mathrm{NMR}$ (Bruker Avance III 400) and FTIR spectrometer (Nicolet iS10 spectrometer, Thermo Fisher Scientific, Waltham, MA, USA). For ${ }^{1} \mathrm{H}-\mathrm{NMR}$ analysis, DOCA was dissolved in $\mathrm{D}_{2} \mathrm{O}$. HES and HESDOCA samples were dissolved in DMSO-d6. For FTIR analysis, HES and HES-DOCA samples were pressed with $\mathrm{KBr}$ and scanned from 4,000 to $400 \mathrm{~cm}^{-1}$ with a resolution of $4 \mathrm{~cm}^{-1}$.

\section{Determination of the substitution degree of HES by DOCA}

The amount of DOCA covalently bounded to HES was determined spectrophotometrically as previously described. ${ }^{15}$ Briefly, the polymer sample (5-10 mg) was accurately weighed and dissolved in $0.5 \mathrm{~mL}$ DMSO. Then, $0.5 \mathrm{~mL}$ acetic acid solution (60\%) and $9.0 \mathrm{~mL}$ water/sulfuric acid $(65 / 50, \mathrm{v} / \mathrm{v})$ were subsequently added. The samples were vortexed to homogeneity, heated at $70^{\circ} \mathrm{C}$ for $30 \mathrm{~min}$ and then cooled to room temperature. The UV (Lambda 35, PerkinElmer Inc., Waltham, MA, USA) absorbance of sample solution was read at $380 \mathrm{~nm}$. Meanwhile, the sample containing the same components without the polymer was used as blank control. Standard calibration curves of DOCA concentration under the abovementioned condition were established. The degree of substitution (DS, mol\%, expressed as mole DOCA/100 sugar residues of HES) was calculated according to the following formula:

$$
\mathrm{DS}=\frac{\left(\mathrm{m}_{\mathrm{DOCA}} / \mathrm{M}_{\mathrm{DOCA}}\right)}{\left[\left(\mathrm{m}_{\mathrm{HES}-\mathrm{DOCA}}-\mathrm{m}_{\mathrm{DOCA}}\right) / \mathrm{M}_{\mathrm{HES}}\right]}
$$

where $\mathrm{m}_{\text {DOCA }}$ is the mass ratio of DOCA in HES-DOCA conjugates calculated from the established calibration curve; $\mathrm{M}_{\mathrm{DOCA}}$ is the molecular weight of the DOCA residue, $\mathrm{m}_{\text {HES-DOCA }}$ is the amount of the HES-DOCA conjugates, $\mathrm{M}_{\mathrm{HES}}$ is the average molecular weight of glucose units of HES.

\section{Preparation of self-assembled HES-DOCA NPs}

To prepare the self-assembled HES-DOCA NPs, $20 \mathrm{mg}$ of HES-DOCA conjugates were dispersed into $2 \mathrm{~mL}$ of acetone and the dispersions were further added dropwise in $4 \mathrm{~mL}$ of phosphate-buffered saline (PBS, $\mathrm{pH}$ 7.4). The mixture was sonicated at $100 \mathrm{w}$ (Automatic Ultrasonic Processor UH-500A, People's Republic of China) in an ice bath for $10 \mathrm{~min}$ followed by stirring at room temperature overnight to remove organic solvents. The resultant mixture was passed through $0.45 \mu \mathrm{m}$ filter membrane to obtain self-assembled HES-DOCA NPs. To prepare the self-assembled HES-DOCA nanoparticles-loaded Morin (Morin/HES-DOCA-NPs), $20 \mathrm{mg}$ of amphiphilic HES-DOCA conjugates and $4 \mathrm{mg}$ Morin were dispersed into $2 \mathrm{~mL}$ of acetone. Then, the dispersion was processed as described previously to obtain Morin/ HES-DOCA-NPs.

\section{Characterization of self-assembled HES-DOCA NPs}

The average particle size and size distribution of the selfassembled NPs were measured in triplicate by photon correlation spectroscopy (Zetasizer Nano ZS90, Malvern Instruments, Malvern, UK). The morphology of NP-loaded Morin was observed by a transmission electron microscopy (TEM) instrument (H-600, Hitachi Ltd., Tokyo, Japan). The TEM specimens were prepared by depositing the NPs suspension onto a copper grid followed by negatively staining with $1 \%$ phosphotungstic acid for $10 \mathrm{~s}$.

Morin concentrations both in vitro and in vivo samples were analyzed on a Waters ${ }^{\circledR} 2690$ HPLC system equipped with a Waters 996 Photodiode Array Detector (Waters, Milford, MA, USA) as we reported previously. ${ }^{16}$ A Zorbax SB-C ${ }_{18}$ analytical column (Agilent, $150 \times 4.6 \mathrm{~mm}^{2}, 5 \mu \mathrm{m}$ ) was used with a mobile phase consisting of acetonitrile and $0.5 \%$ phosphoric acid $(24: 76, \mathrm{v} / \mathrm{v})$ with a flow rate of $1.0 \mathrm{~mL} / \mathrm{min}$. The detection wavelength was $252 \mathrm{~nm}$. The DL and encapsulation efficiency (EE) were calculated by the following equations:

$$
\begin{gathered}
\mathrm{DL} \%=\frac{\text { Weight of the drug in nanoparticles }}{\text { Weight of the feeding polymer and drug }} \times 100 \% \\
\mathrm{EE} \%=\frac{\text { Weight of the drug in nanoparticles }}{\text { Weight of the feeding drug }} \times 100 \%
\end{gathered}
$$

\section{In vitro release of Morin/HES-DOCA-NPs}

In vitro release studies were performed using a dialysis method in phosphate buffer solutions $\left(\mathrm{pH} \mathrm{7.4)}\right.$ at $37^{\circ} \mathrm{C}$. 
The dialysis bags (MWCO of 3500) were first soaked in medium for $12 \mathrm{~h}$ before use. In total, $1 \mathrm{~mL}$ of freshly prepared Morin solution or Morin/HES-DOCA-NPs (diluted with release medium, $0.5 \mathrm{mg} / \mathrm{mL}$ ) was added to the bags, which were placed in antibiotic bottles containing $50 \mathrm{~mL}$ of the release medium and shaken at $100 \mathrm{rpm}$. At designated time points, $1 \mathrm{~mL}$ of the release medium was withdrawn, and replaced by an equal volume of fresh media. The samples collected were analyzed using HPLC as described previously.

\section{In vivo pharmacokinetic studies}

Rats were randomly divided into two groups $(n=5)$ and fasted overnight before administration. One group was given intravenously Morin saline solution at $2 \mathrm{mg} / \mathrm{kg}$. The other group was given Morin/HES-DOCA-NPs at an equivalent dose of $2 \mathrm{mg} / \mathrm{kg}$. Blood $(400 \mu \mathrm{L})$ was collected via orbital sinus puncture into heparinized tubes at designated time points post injection. Plasma was separated from whole blood by centrifugation $(1,296 \times g, 5 \mathrm{~min})$. The sample procession was adopted from previous work. ${ }^{10}$ Briefly, rat plasma $(100 \mu \mathrm{L})$ was mixed with $100 \mu \mathrm{L} 25 \%$ hydrochloric acid and $200 \mu \mathrm{L}$ of methanol containing $30 \mu \mathrm{g} / \mathrm{mL}$ benzoic acid as internal standard. The mixture was further incubated in $50^{\circ} \mathrm{C}$ water bath for $0.5 \mathrm{~h}$. After centrifugation $(12,544 \times \mathrm{g}$, $15 \mathrm{~min})$, the supernatant $(100 \mu \mathrm{L})$ was injected directly into the HPLC column.

The pharmacokinetic parameters, such as the area under the plasma concentration-time curve (AUC), elimination half-life and mean residence time were determined by DAS 3.0 software (Mathematical Pharmacology Professional Committee of China).

\section{Animal model of hyperuricemic rats and drug administration}

A hyperuricemia model in rats was established using uricase inhibitor potassium oxonate as we described previously. ${ }^{9}$ Rats were randomly assigned to four groups $(n=10)$ as follows: normal group; hyperuricemic control group; Morin-treated group and Morin/HES-DOCA-NPs-treated group. Prior to experimental procedure, blank serum of all these four groups was collected via orbital puncture. Then potassium oxonate suspension in $0.5 \%$ CMC-Na was intraperitoneally injected to rats at the dose of $250 \mathrm{mg} / \mathrm{kg}$, while normal saline of similar volume was injected into each rat of the normal group. Blood samples were collected from each rat $1 \mathrm{~h}$ postinjection. Thereafter, the induced hyperuricemic rats were intravenously given Morin solutions and Morin/HES-DOCA-NPs at an equivalent dose of $2 \mathrm{mg} / \mathrm{kg}$ Morin, respectively. The normal and hyperuricemic control group (non-treated) were intravenously given a similar volume of normal saline. For each group, $0.5 \mathrm{~mL}$ of plasma was collected via orbital puncture at designated time points and centrifuged at $10,000 \times g$ for 5 min to obtain serum. The serum uric acid, creatine and urea nitrogen levels of the samples were determined by an automatic biochemical analyzer using the corresponding kit (Hitachi 7020). In the end, kidneys were separated from sacrificed rats and fixed in $4 \%$ buffered formalin. Each was then paraffin embedded and sectioned for hematoxylin and eosin staining for conventional histological examination. Photomicrographs were taken with a microscope camera at $200 \times$ magnification.

\section{Determination of uric acid excretion, hepatic and serum xanthine dehydrogenase/xanthine oxidase $(\mathrm{XDH} / \mathrm{XO})$ activity in hyperuricemic rats}

Rats were housed individually in metabolic cages and allowed free access to water during the period of the experiment. After the abovementioned procedures, urine was collected for $5 \mathrm{~h}$ after each drug treatment. Thereafter, blood samples of the animals were collected by orbital puncture. The uric acid and creatinine levels in serum of the animals were determined using the automatic biochemical analyzer. After being killed by decapitation, the liver tissues of rats were dissected rapidly and were homogenized in 10 volumes of ice-cold saline. After centrifugation $(6,000 \times g, 10 \mathrm{~min})$, the $\mathrm{XDH} / \mathrm{XO}$ activity of the supernatant was determined using a spectrophotometric method by $\mathrm{XDH} / \mathrm{XO}$ assay kits (Nanjing Jiancheng Co., People's Republic of China). $\mathrm{XDH} / \mathrm{XO}$ activity was expressed as per $\mu \mathrm{moL}$ xanthine yielded per $g$ per minute of tissue protein.

Urine uric acid concentration was analyzed by HPLC as described in our earlier studies. ${ }^{9}$ Urine creatine levels were determined by the automatic biochemical analyzer. The fractional excretion of urate $\left(\mathrm{FE}_{\mathrm{UA}}\right)$ was calculated by the following equation:

$$
\mathrm{FE}_{\mathrm{UA}}=\frac{\text { Urine urate } \times \text { Plasma creatine }}{\text { Urine creatine } \times \text { Plasma urate }}
$$

\section{Statistical analysis}

Data were assessed by two-tailed Student's $t$-test or one-way ANOVA followed by Turkey's multiple comparison tests. A $P$-value of less than 0.05 was considered significant. 


\section{Results \\ Synthesis and characterization of HES- DOCA conjugates}

Scheme 1A illustrates the detailed chemical structure and synthesis procedures of HES-DOCA conjugates. Hydrophobically modified HES polymer was prepared by covalent attachment of DOCA to HES through ester bond, producing a novel kind of amphiphilic polymer. The structure of resulting HES-based polymer was confirmed by ${ }^{1} \mathrm{H}-\mathrm{NMR}$ and FTIR, as shown in Figure 1. As revealed by ${ }^{1} \mathrm{H}-\mathrm{NMR}$ spectrum (Figure 1A), the characteristic peaks of HES located between 4.5 and $5.6 \mathrm{ppm}$, which could be assigned to the hydroxyl groups from glucose unit of HES. ${ }^{17,18}$ Successful introduction of DOCA into HES polymers was validated by the peaks of DOCA appearing at $0.6-2.5 \mathrm{ppm} .{ }^{19}$ The formation of ester linkage between DOCA and HES was also evidenced in the FTIR spectra (Figure 1B). The characteristic peak of DOCA at 2,936 and 2,862 $\mathrm{cm}^{-1}$ [Figure 1B (a)] was assigned to the stretching vibration of DOCA..$^{15} \mathrm{~A}$ significant shift at $1,687 \mathrm{~cm}^{-1}$ was the carbonyl band of DOCA units. The bands at 3,428 and $2,934 \mathrm{~cm}^{-1}$ in the spectrum of HES [Figure 1B (b)] were characteristic of $\mathrm{OH}$ and $\mathrm{CH}$ stretches in the HES, respectively. ${ }^{13}$ In comparison with the FTIR spectra of HES and DOCA, the new absorption band at $1,714 \mathrm{~cm}^{-1}$ [Figure 1B (c)] was assigned to the carbonyl of ester bond of HES-DOCA conjugates, further confirming the formation of HES-DOCA conjugates.

By controlling the feed ratio of DOCA to HES from 25 to $200 \%$, the DS of DOCA ranged from 1.2 to 35.4 , as shown in Table 1. The mean size of self-assembled NPs of HES-DOCA conjugates with different DS was in the range of 159 and $235 \mathrm{~nm}$ in PBS solution (pH 7.4) as determined by dynamic light scattering (DLS). The polydispersity index was less than 0.2 for all the prepared HES-DOCA NPs, indicated that the HES-DOCA NPs formed in PBS solution were monodispersed with narrow particle size distribution. Zeta potential indicated that HES-DOCA NPs were covered with negatively charged HES shells from -7 to $-18.9 \mathrm{mV}$, leading to a repelling force between particles with increased stability. Considering the optimal size of NPs for drug delivery, the DS value was controlled around $10 \%$ for HES-DOCA conjugates in the following formulation studies. Using the pyrene-based fluorescence technique, the critical aggregation concentration of HESDOCA conjugates with DS of $10 \%$ was determined as $0.0215 \mathrm{mg} / \mathrm{mL}$.

A
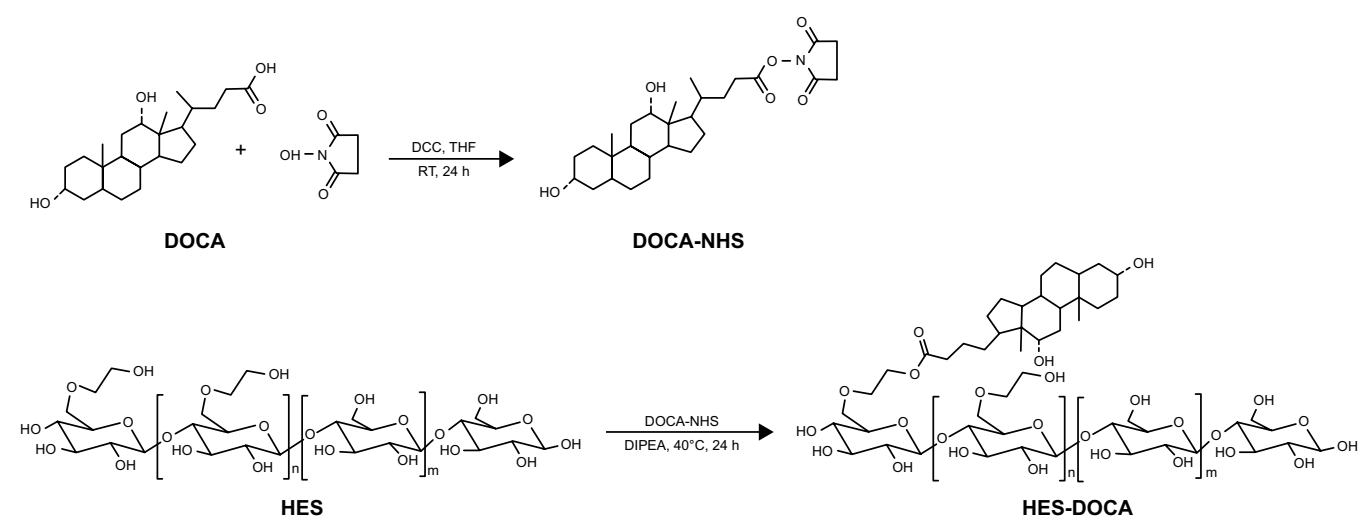

B
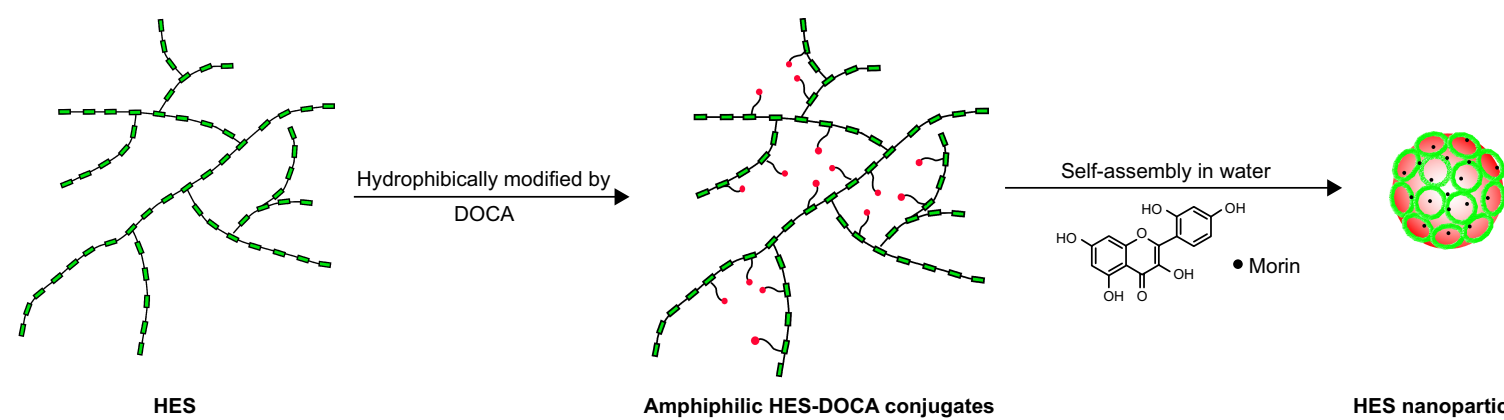

$\square$ Glucose or hydroxyethyl glucose $\bigcirc$ DOCA

Scheme I The synthesis scheme of HES-DOCA conjugates (A) and schematic illustration of DOCA-mediated self-assembled HES-based nanoparticles (B). Abbreviations: HES, hydroxyethyl starch; DOCA, deoxycholic acid; HES-DOCA, hydroxyethyl starch-deoxycholic acid. 
A

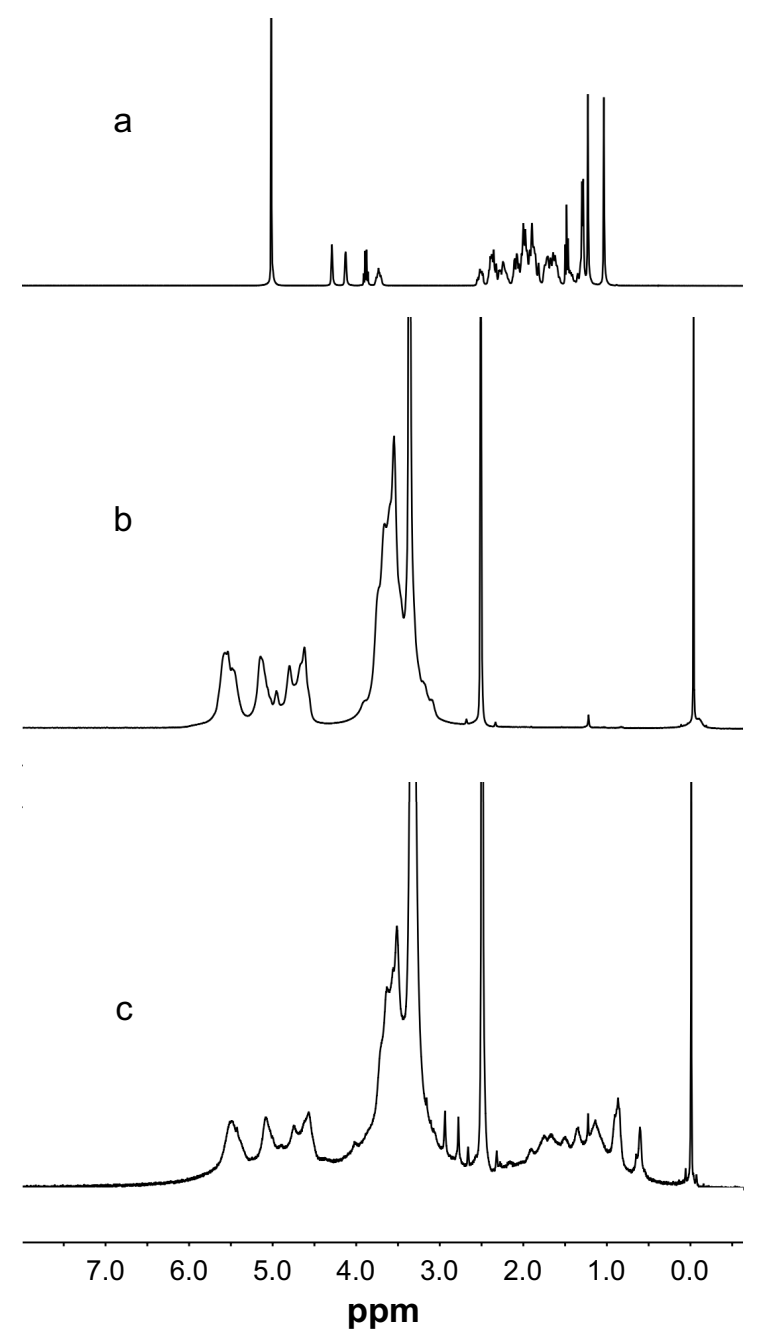

B

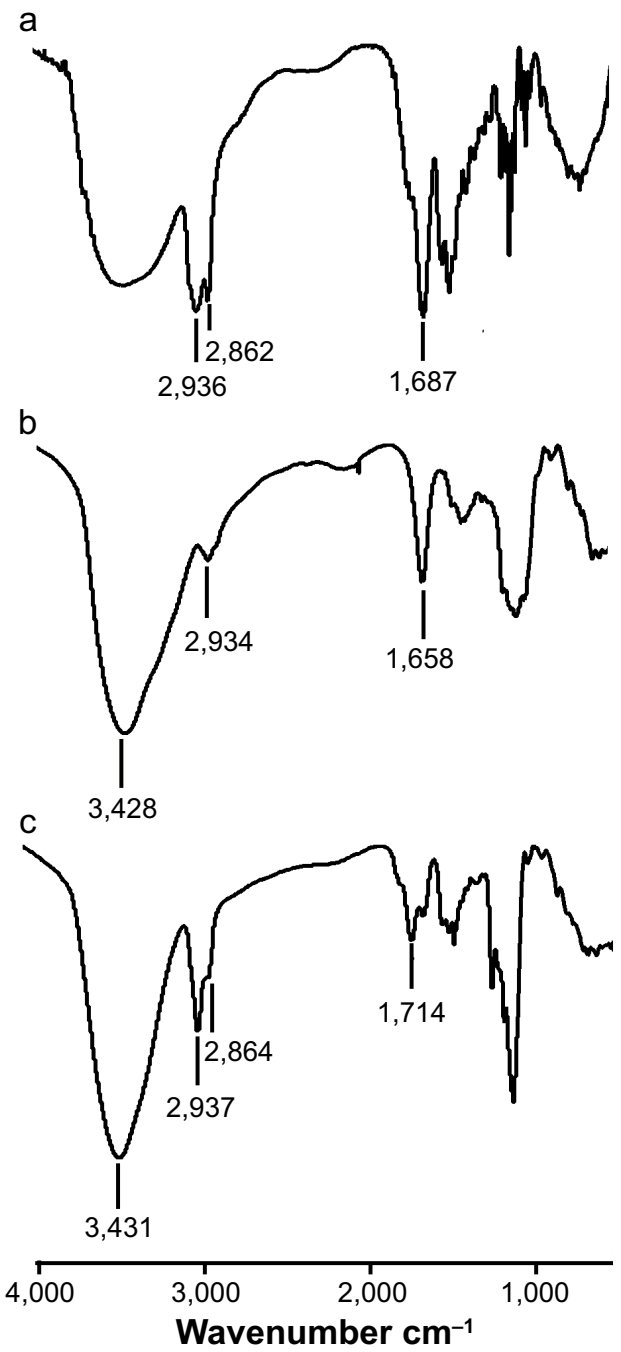

Figure I (A) 'H-NMR spectrum of (a) DOCA, (b) HES and (c) HES-DOCA; (B) FTIR spectra of (a) DOCA, (b) HES and (c) HES-DOCA. Abbreviations: HES, hydroxyethyl starch; DOCA, deoxycholic acid; HES-DOCA, hydroxyethyl starch-deoxycholic acid.

Table I Characteristics of HES-DOCA conjugates in PBS solution $(\mathrm{pH} 7.4)$

\begin{tabular}{lllll}
\hline Sample & $\begin{array}{l}\text { Feed } \\
\text { ratio }^{\mathbf{a}}\end{array}$ & $\begin{array}{l}\mathbf{D S}^{\mathrm{b}} \\
(\%)\end{array}$ & $\begin{array}{l}\text { Size }^{\mathrm{c}}(\mathbf{n m}) \\
\left(\mu_{\mathbf{2}} / \Gamma^{2, \mathrm{~d}}\right)\end{array}$ & $\begin{array}{l}\text { Zeta potential }^{\mathbf{e}} \\
(\mathbf{m V})\end{array}$ \\
\hline HES-DOCA & $0.25: 1$ & 1.2 & $23 \mathrm{I} \pm 7.3(0.032)$ & -18.9 \\
HES-DOCA & $0.5: 1$ & 2.5 & $175 \pm 4.7(0.109)$ & -19.3 \\
HES-DOCA & $0.75: 1$ & 10.2 & $159 \pm 3.2(0.08 I)$ & -16.3 \\
HES-DOCA & $1: 1$ & 15.7 & $201 \pm 5.1(0.026)$ & -13.4 \\
HES-DOCA & $1.5: 1$ & 29.3 & $23 \mathrm{I} \pm 2.9(0.032)$ & -12.7 \\
HES-DOCA & $1.75: 1$ & 32.1 & $229 \pm 4.3(0.032)$ & -11 \\
HES-DOCA & $2: 1$ & 35.4 & $235 \pm 5.2(0.068)$ & -7 \\
\hline
\end{tabular}

Notes: aMolar feed ratio of DOCA-NHS to hydroxyethyl sugar residues of HES polymer; 'substitution degree of DOCA per 100 anhydroglucose unit; 'mean diameters of nanoparticles measured by dynamic light scattering; ${ }^{\mathrm{d}}$ polydispersity index of nanoparticles size; 'the potential of HES-DOCA conjugates in PBS solution $(\mathrm{pH}=7.4)$ at I $\mathrm{mg} / \mathrm{mL}$.

Abbreviations: HES-DOCA, hydroxyethyl starch-deoxycholic acid; PBS, phosphatebuffered saline; DS, degree of substitution; NHS, N-hydroxysuccinimide.

\section{Preparation and characterization of Morin/HES-DOCA-NPs}

Morin was easily encapsulated into HES-DOCA NPs using a simple combination of solvent evaporation and sonication approaches. The brush-like structured core of NPs was self-aggregated by hydrophobic effects of DOCA, while the hydrophilic HES backbone comprised the shell of NPs, as shown in Scheme 1B. DLS measurement demonstrated that the mean particle size of Morin/HES-DOCA-NPs was $197 \mathrm{~nm}$ with a polydispersity index value of 0.05 and a zeta potential of $-14 \mathrm{mV}$ (Figure 2A). TEM results showed that self-assembled HES NPs were spherical, and their sizes were smaller than the results determined by DLS measurement (Figure 2B). The DL was $92.5 \%$ and the EE was $15.6 \%$. 
A

$\begin{array}{rllll} & & \text { Size (d.nm) } & \text { \% Intensity: } & \text { St dev (d.nm) } \\ \text { Z-average (d, nm): } 197.3 & \text { Peak 1: } & 211.6 & 100.0 & 59.03 \\ \text { Pdl: } 0.050 & \text { Peak 2: } & 0.000 & 0.0 & 0.000 \\ \text { Intercept: } 0.917 & \text { Peak 3: } & 0.000 & 0.0 & 0.000\end{array}$

Result quality Good

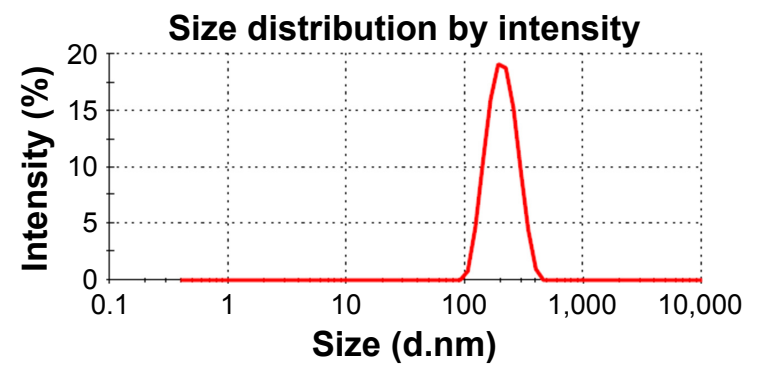

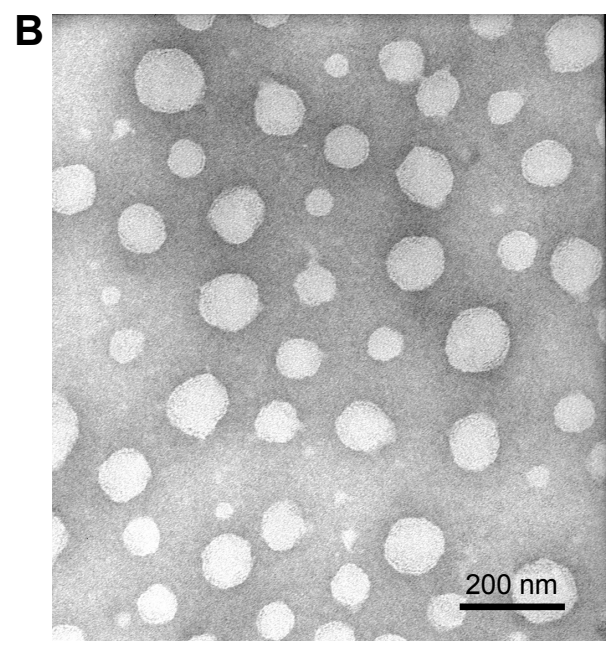

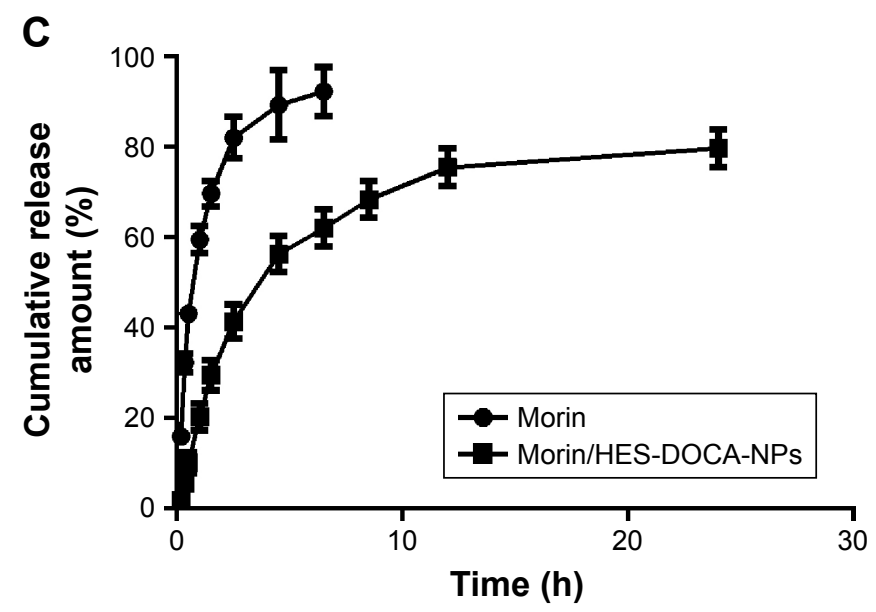

Figure 2 Characterization of Morin/HES-DOCA-NPs. (A) Particle size distribution of Morin/HES-DOCA-NPs; (B) negative-stain TEM image of Morin/HES-DOCA-NPs and (C) drug release profiles from Morin/HES-DOCA-NPs in PBS solution $(\mathrm{pH} 7.4)$ at $37^{\circ} \mathrm{C}$.

Abbreviations: Morin/HES-DOCA-NPs, hydroxyethyl starch-deoxycholic acid nanoparticles-loaded Morin; TEM, transmission electron microscopy; PBS, phosphatebuffered saline; St dev, standard deviation.

The in vitro release profile of Morin from HES-DOCA conjugates in PBS solution ( $\mathrm{pH}$ 7.4) is shown in Figure 2C. Free Morin released quickly in PBS solution, which was also demonstrated in our previous study, probably due to the better solubility of free Morin at higher $\mathrm{pH}$ values. ${ }^{16}$ By contrast, Morin was gradually released from HES-DOCA NPs over an extended period. No obvious burst release effects were observed in Morin/HES-DOCA-NPs, indicating Morin was loaded mainly in the hydrophobic core of HES-DOCA NPs but was not physically adsorbed onto its hydrophilic outer shell.

\section{Pharmacokinetic of Morin/HES-DOCA-}

\section{NPs after intravenous administration}

The mean plasma concentration-time curves of Morin and Morin/HES-DOCA-NPs via tail vein injection are shown in Figure 3. Compared with Morin, Morin/HES-DOCA-NPs

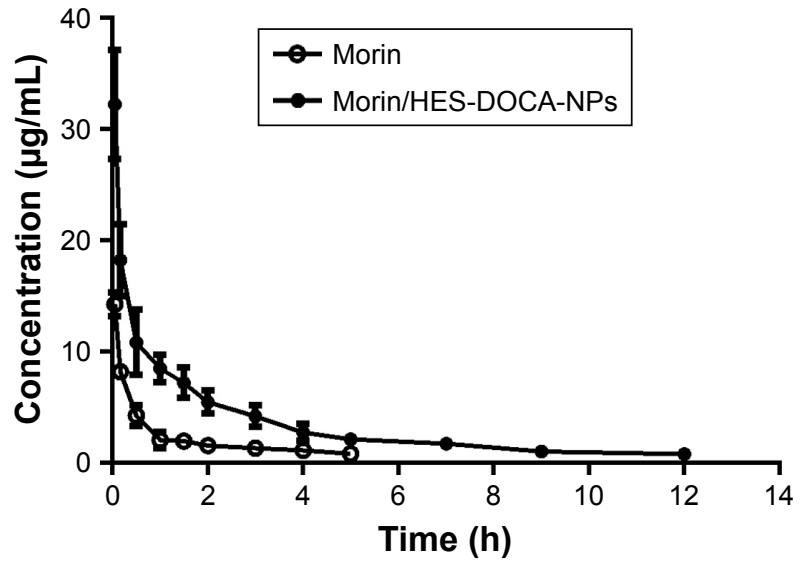

Figure 3 Mean plasma concentration-time curves after intravenous administration of Morin and Morin/HES-DOCA-NPs (at a dose of $2 \mathrm{mg} / \mathrm{kg}$, equivalent to free Morin treatment) in Wistar rats.

Note: Data are expressed as mean \pm standard deviation $(n=5)$.

Abbreviation: Morin/HES-DOCA-NPs, hydroxyethyl starch-deoxycholic acid nanoparticles-loaded Morin. 
exhibited remarkably higher plasma level and extended clearance time from the plasma after injection in rats. Specifically, the plasma concentrations of Morin were significantly higher in rats administered with Morin/HES-DOCA-NPs than those administered with Morin solution at all time points postinjection. In addition, free Morin was rapidly eliminated from plasma and hardly detected $5 \mathrm{~h}$ after administration of Morin solution. By contrast, Morin/HES-DOCA-NPs demonstrated significantly longer blood-circulation time than free Morin as Morin was still detectable $12 \mathrm{~h}$ postinjection. The relevant pharmacokinetic parameters were obtained using a two compartmental analysis (Table 2). $\mathrm{AUC}_{0-\mathrm{t}}$ values were increased to 2.6 times in Morin/HES-DOCA-NPs formulations, compared to free Morin. Remarkably, the half-life of Morin $(0.37 \mathrm{~h})$ was significantly enhanced by more than fourfold by the incorporation of Morin into HES-DOCA NPs (1.52 h). These encouraging results suggest a possible higher therapeutic effect of Morin/HES-DOCA-NPs compared with Morin solution.

\section{Reduced serum uric acid level by Morin/ HES-DOCA-NPs in hyperuricemic rats}

Hyperuricemia group, Morin-treated group and Morin/ HES-DOCA-NPs-treated group demonstrated a significant increase in the serum uric acid level of rats at $2 \mathrm{~h}$, in comparison with blank group (Figure 4A). Both Morin and Morin/HES-DOCA-NPs treatment reduced the serum uric acid level at $4 \mathrm{~h}$, compared to hyperuricemia group (control), but only Morin/HES-DOCA-NPs further reduced serum uric acid level to normal $(55 \mu \mathrm{mol} / \mathrm{L})$ at $6 \mathrm{~h}$, indicating its superior antihyperuricemic efficacy (Figure 4B). Although it is not statistically significant, obvious trends in increasing the serum creatine and nitrogen levels were present between hyperuricemia group and blank group (Figure 4C and D), suggesting possible renal injury caused by potassium oxonate..$^{20,21}$

Table 2 Pharmacokinetic parameters of Morin in rats after the intravenous injection of Morin and Morin/HES-DOCA-NPs at a dose of $2 \mathrm{mg} / \mathrm{kg}(\mathrm{n}=5)$

\begin{tabular}{lll}
\hline Formulations & Morin & $\begin{array}{l}\text { Morin/HES- } \\
\text { DOCA-NPs }\end{array}$ \\
\hline AUC $_{0-\mathrm{t}}(\mathrm{mg} / \mathrm{L} \mathrm{h})$ & $20.89 \pm 3.54$ & $54.39 \pm 6.07^{* *}$ \\
$\mathrm{MRT}(\mathrm{h})$ & $1.23 \pm 0.32$ & $3.91 \pm 0.49 *$ \\
$\mathrm{~T}_{1 / 2 \beta}(\mathrm{h})$ & $0.37 \pm 0.02$ & $1.52 \pm 0.25^{*}$ \\
$\mathrm{C}_{\max }(\mu \mathrm{g} / \mathrm{mL})$ & $14.27 \pm 2.57$ & $32.24 \pm 3.2 \mathrm{I}^{*}$ \\
\hline
\end{tabular}

Notes: $* P<0.05$ compared with Morin group; $* * P<0.01$, compared with Morin group.

Abbreviations: Morin/HES-DOCA-NPs, hydroxyethyl starch-deoxycholic acid nanoparticles-loaded Morin; $\mathrm{AUC}_{0-\mathrm{t}}$, area under the concentration-time curve from time zero to the last sampling time point; MRT, mean residence time; $T_{1 / 2 \beta}$, elimination half-life; $C_{\max }$, maximum plasma concentration of Morin.
Compared to hyperuricemia rats, Morin/HES-DOCA-NPs treatment demonstrated a marked decrease in the serum creatine and nitrogen levels, which was not observed for Morin treatment. The results suggested that Morin/HESDOCA-NPs might have protective effects against kidney damage in hyperuricemia rats. As shown in Figure 4E, hyperuricemia group (control) demonstrated significant morphological changes in renal tissue typically characterized by inflammatory cell infiltration, compared with normal rats. Morin-treated groups showed alleviated inflammatory cell influx in the tubule and collecting duct of rats, suggesting that Morin could slightly attenuate rat kidney damage. By contrast, renal tissue in Morin/HES-DOCA-NPs-treated rats preserved normal kidney structure, with minimal morphological changes. Therefore, Morin/HES-DOCA-NPs had superior ability in protecting rat kidney from acute injury than Morin.

\section{Uricosuric effects and inhibitory effect of Morin/HES-DOCA-NPs on hepatic $\mathrm{XDH} / \mathrm{XO}$ activity in hyperuricemic rats}

Both serum and hepatic XDH/XO activity of rats in these four groups were determined to evaluate the inhibitory effect of drugs on production of uric acid in vivo. As shown in Figure 5, no significant difference was observed among the serum $\mathrm{XDH} / \mathrm{XO}$ activities of these four groups (Figure $5 \mathrm{~A}$ ). Figure 5B demonstrates that Morin/HES-DOCA-NPs significantly decreased $\mathrm{XDH} / \mathrm{XO}$ activity in liver in comparison with Morin, suggesting the vital role of hepatic XDH/XO activity in modulating uric acid level by Morin/HES-DOCA-NPs. The urine uric acid level was significantly increased by 3.6-fold in the hyperuricemia control rat (without drug treatment) compared with normal rats (Figure S1), further confirming that the hyperuricemic rat kidney was injured soon after uric acid level increased. Compared with Morin, Morin/ HES-DOCA-NPs significantly increased urine uric acid level of rats, suggesting the superior uricosuric action of Morin/ HES-DOCA-NPs. Moreover, UA excretion was expressed as fractional excretion $\left(\mathrm{FE}_{\mathrm{UA}}\right)$. All the other parameters, including urine and plasma creatine levels and urine volume (Figure S1A-C), were measured for the four groups of rats to calculate $\mathrm{FE}_{\mathrm{UA}}$. Both Morin and Morin/HES-DOCANPs-treated rats demonstrated significantly higher $\mathrm{FE}_{\mathrm{UA}}$ values than the normal group (Figure $5 \mathrm{C}$ ). The $\mathrm{FE}_{\mathrm{UA}}$ value of Morin/HES-DOCA-NPs (0.64) was four times that of Morin (0.16), which further confirmed that Morin/HES-DOCA-NPs significantly improved the uricosuric actions of Morin, contributing to the improved antihyperuricemic effect in rats. 
A

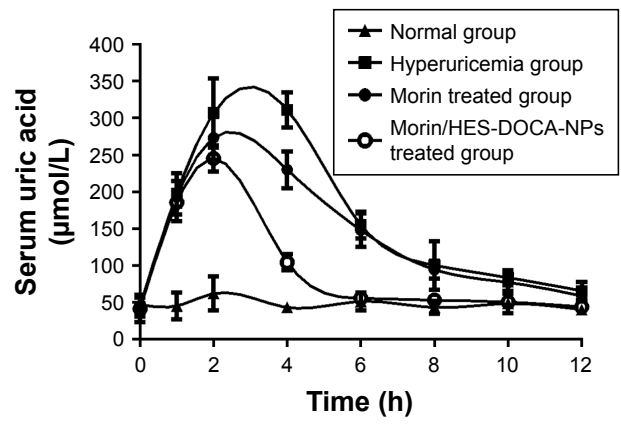

C

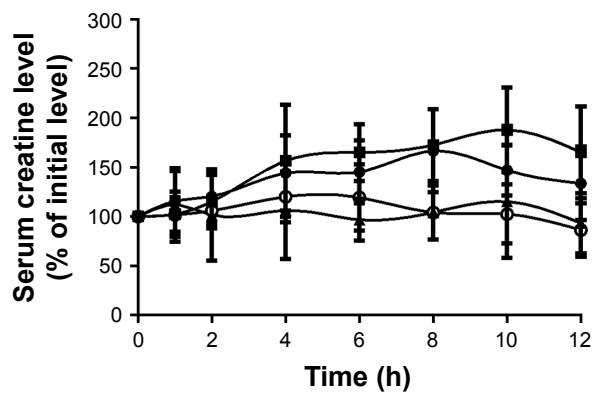

B

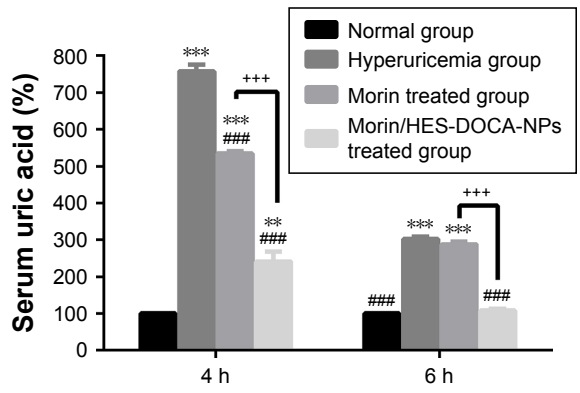

D

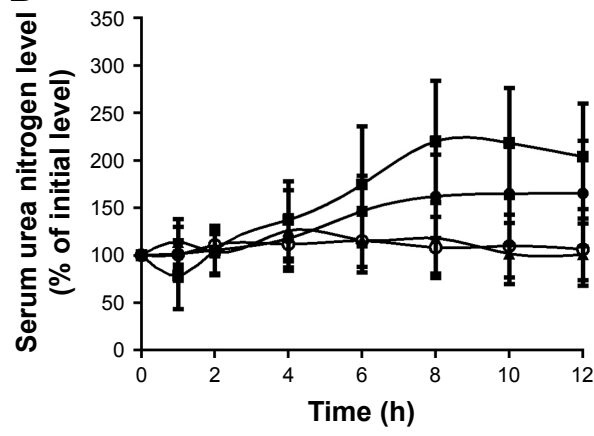

$\leftarrow$ Normal group $\rightarrow$ Hyperuricemia group $\rightarrow$ Morin treated group $\multimap-$ Morin/HES-DOCA-NPs treated group

E
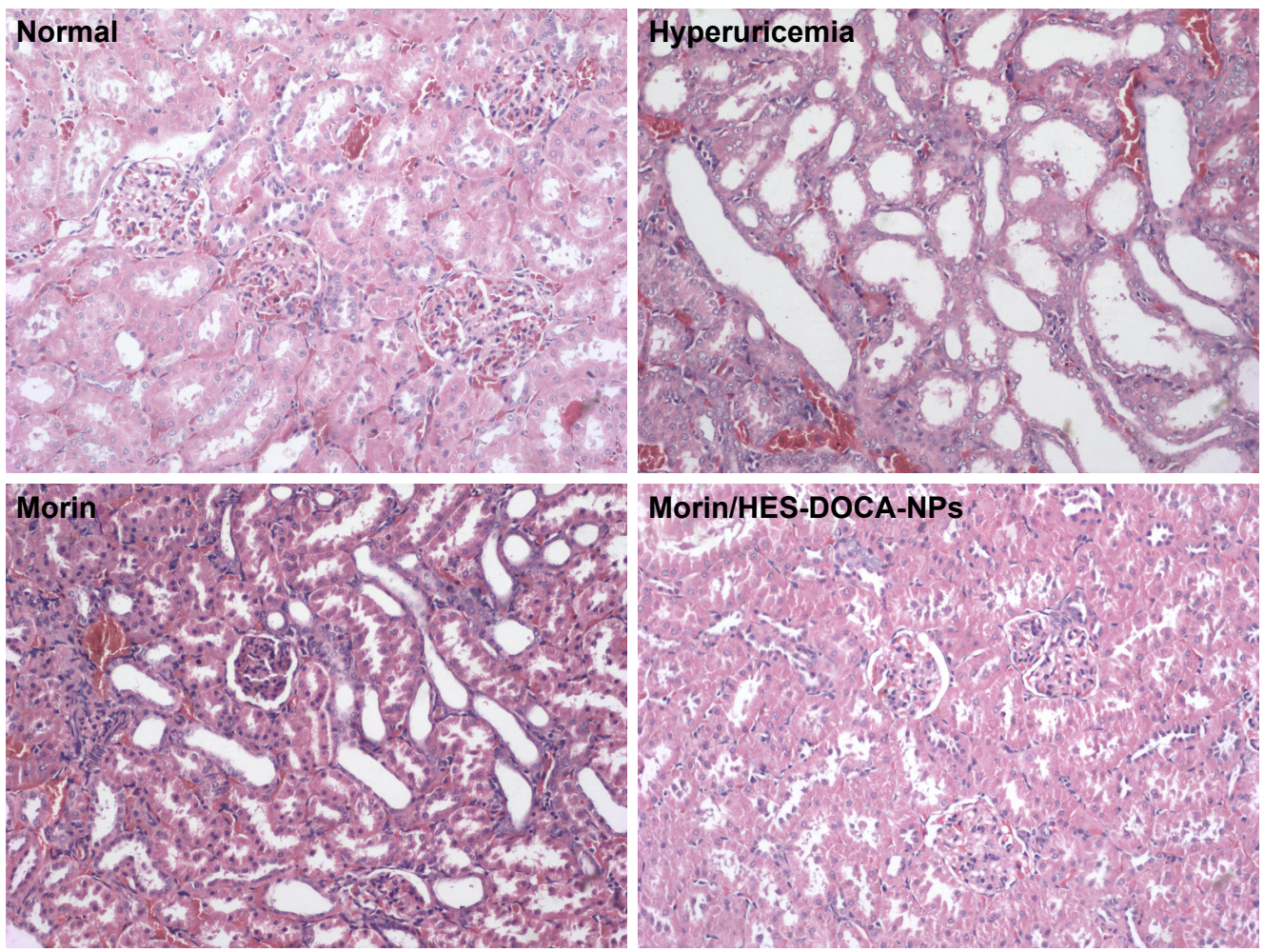

Figure 4 Therapeutic efficacy of Morin/HES-DOCA-NPs on oxonate potassium-induced hyperuricemia rats. (A) Serum uric acid level-time curves of rats under different treatment; (B) serum uric acid level of oxonate potassium-induced hyperuricemia rats after administration of Morin and Morin/HES-DOCA-NPs. The serum uric acid level of normal rats was set as $100 \%$. Notations were the same as stated in (A). (C) Effect of Morin/HES-DOCA-NPs on serum creatine level of hyperuricemia rats; (D) effect of Morin/HES-DOCA-NPs on serum urea nitrogen level of hyperuricemia rats; (E) histological evaluation of rats kidney under different treatment.

Notes: Normal group: rats without any treatment; Hyperuricemia group: given an intraperitoneal injection of potassium oxonate (250 mg/kg) at 0 h; Morin and Morin/HESDOCA-NPs-treated group: intravenously given Morin solution and Morin/HES-DOCA-NPs at $2 \mathrm{mg} / \mathrm{kg}$, respectively, $\mathrm{I} \mathrm{h}$ after intraperitoneal injection of potassium oxonate.

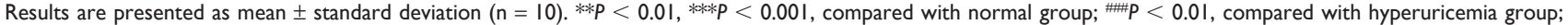
$++P<0.001$, compared with Morin treated group. Magnification: $200 \times(E)$.

Abbreviation: Morin/HES-DOCA-NPs, hydroxyethyl starch-deoxycholic acid nanoparticles-loaded Morin. 
A

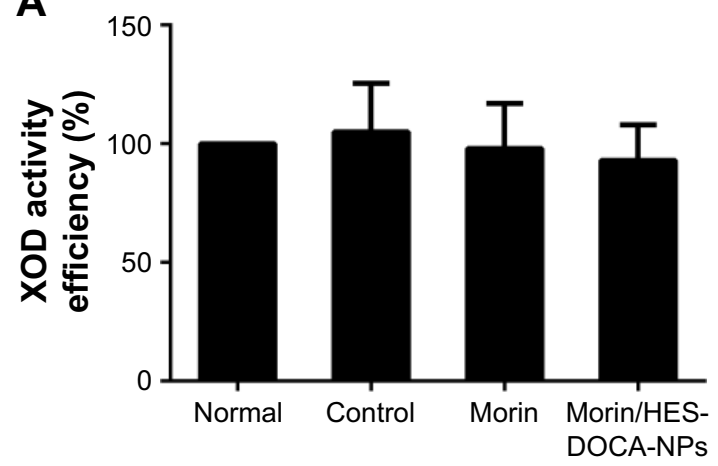

B

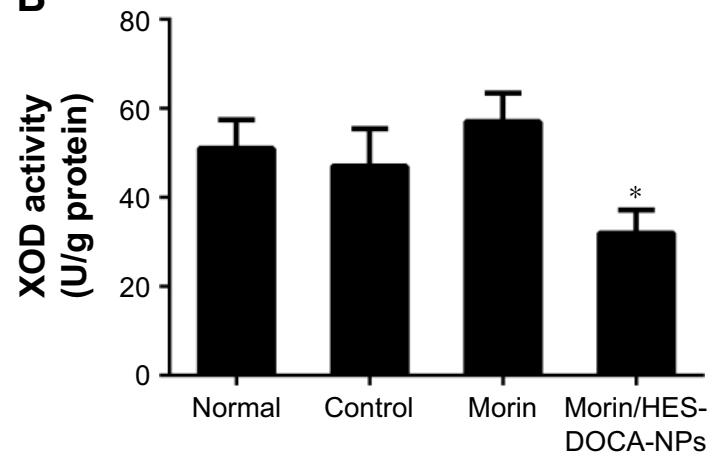

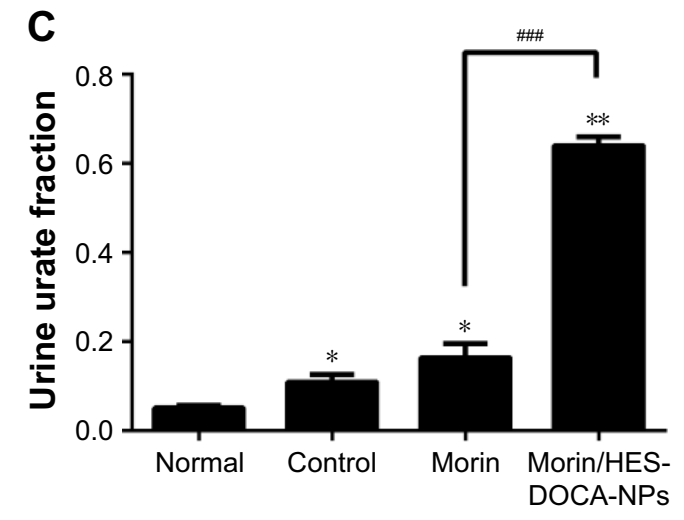

Figure 5 Therapeutic mechanism of Morin/HES-DOCA-NPs on decreasing serum uric acid level in vivo. Inhibition effect of Morin/HES-DOCA-NPs on xanthine oxidase activity in serum (A) and liver (B) of rats under different conditions. Both serum and liver samples were obtained after intraperitoneal injection of potassium oxonate for $5 \mathrm{~h}$. In vivo uricosuric actions of Morin and Morin/HES-DOCA-NPs on hyperuricemic rats induced by potassium oxonate (C). Urine was collected for 5 h.

Notes: Normal: rats without any treatment; Control (Hyperuricemia group): given an intraperitoneal injection of potassium oxonate (250 mg/kg) at 0 h; Morin and Morin/ HES-DOCA-NPs group: intravenously given Morin solution and Morin/HES-DOCA-NPs at $2 \mathrm{mg} / \mathrm{kg}$, respectively, I h after intraperitoneal injection of potassium oxonate. Results are presented as mean \pm standard deviation $(n=10)$. $* P<0.05$, **P $<0.01$, compared to normal group. ${ }^{\#} P<0.00$ I, compared with Morin group. Abbreviation: Morin/HES-DOCA-NPs, hydroxyethyl starch-deoxycholic acid nanoparticles-loaded Morin.

\section{Discussion}

With an ever-growing aging population and greatly improved living conditions, hyperuricemia has placed a significant burden on the public healthcare system..$^{22}$ Morin is one of the medicinally important flavonoids and exhibits not only inhibitory activity against xanthine oxidase but also potent inhibitory action on urate uptake in rat renal brush-border membrane vesicles, indicating its great potential in regulating uric acid level in vivo. ${ }^{7}$ Its poor water solubility and short plasma half-life, however, have prevented its clinical use in the treatment of hyperuricemia. ${ }^{9,10}$

To overcome these limitations, we designed an amphiphilic HES-based polymer by conjugating DOCA covalently to the hydroxyl ethyl group of HES. In addition to induce stable HES-based self-assembled NPs, DOCA was also expected to serve as a microcontainer for high DL of poor water-soluble Morin, due to its distinctive amphiphilicity. ${ }^{19}$ The outer shell comprising HES with free hydroxyl ethyl groups was expected to display stealth properties in systemic circulation to prolong the half-life of encapsulated Morin and further improve its therapeutic efficacy.
More importantly, from clinical translational perspective, the HES-based amphiphilic polymer design and its NP formulation have significant advantages. HES is currently intensely investigated as a substitute for PEGs. ${ }^{17}$ Compared with synthetic PEGs, naturally derived HES shows negligible toxicity and immunity to the body, which opens a new door for exploring in vivo application of naturally derived materials with stealth properties. ${ }^{23}$ However, very few studies have reported the design, synthesis and in vivo evaluation of HES-based amphiphilic polymer. ${ }^{12}$ Our study is the first to explore the long circulation properties of HES-based nanoplatforms and validate its utility in drug delivery for flavonoid Morin as well as clinically relevant hyperuricemia model.

In this study, we have successfully synthesized HESDOCA conjugates with different DS of DOCA and the structure was confirmed by analysis of FTIR and ${ }^{1} \mathrm{H}-\mathrm{NMR}$. As expected, the DS increased as the feed ratio of DOCANHSE to the hydroxyethyl sugar residues of HES polymer increased. Interestingly, the mean sizes of self-assembled NPs decreased from 231 to $159 \mathrm{~nm}$ when DS of HESDOCA conjugates increased from $1.2 \%$ to $10 \%$, and then 
the mean sizes increased to $235 \mathrm{~nm}$ when DS increased to $35 \%$. Based on our preliminary study, water-soluble HES has a mean diameter of about $230 \mathrm{~nm}$ in water, probably due to its dendrimer-like properties. Compared with native HES, the size of self-assembled HES-DOCA NPs (DS, $1.2 \%-10 \%$ ) slightly decreased mainly due to the hydrophobic force-mediated compaction of HES by DOCA. When DS increased over $10 \%$, the sizes of self-assembled HES-DOCA NPs began to increase probably because of high density of DOCA conjugation-induced larger hydrophobic inner core. Considering both optimal size and suitable DS of DOCA for drug delivery, the HES-DOCA conjugate with a DS of $10 \%$ was selected for the following studies. The selected HESDOCA conjugates have a mean size of $159 \mathrm{~nm}$ when selfassembled into NPs and its CMC value was determined to be $0.0215 \mathrm{mg} / \mathrm{mL}$, suggesting that the NPs are stable and may have the capacity to retain their aggregation in the systemic circulation. When formulated with free drug Morin, the mean size of HES-DOCA NPs (DS 10\%) increased to $197 \mathrm{~nm}$ and exhibited a zeta potential of $-14 \mathrm{mV}$. The size measured by DLS was slightly larger than the results determined by TEM. It was mainly due to the solvent effect of different state of samples. DLS showed the size for the particles swollen in the solution, while TEM images showed the size at the dried state of the samples. ${ }^{15}$

In vitro release study showed sustained release of Morin from HES-DOCA NPs for $24 \mathrm{~h}$, confirming the stable encapsulation and ability of NPs to extend the availability of Morin in blood circulation. In vivo pharmacokinetics data support the long circulation of Morin in blood as the half-life of Morin increased by threefold after Morin/HES-DOCA-NPs administration, compared with Morin solution.

The improved therapeutic index of Morin/HES-DOCANPs was validated in an oxonate potassium-induced hyperuricemic rat model. Morin/HES-DOCA-NPs significantly reduced the high serum uric acid level of hyperuricemic rat ( $145 \mu \mathrm{mol} / \mathrm{L})$ to normal $(55 \mu \mathrm{mol} / \mathrm{L}) 5 \mathrm{~h}$ post-administration (Figure 4A). By contrast, the antihyperuricemic effect of Morin diminished after Morin solution was given intravenously for $5 \mathrm{~h}$, probably due to the short half-life of free Morin in vivo.

The histological examination revealed that the kidney of hyperuricemic rats was injured soon after uric acid level increased, in consistent with previous reports. ${ }^{24}$ Morin was previously reported to possess anti-inflammatory effect but showed minimal protective effect against renal inflammation in hyperuricemic rats. ${ }^{6}$ It may be due to the insufficient serum level of Morin at the dose of $2 \mathrm{mg} / \mathrm{kg}$ for satisfied therapeutic efficacy. In a previous study, we explored the therapeutic mechanism of Morin in vivo and found that Morin inhibited $\mathrm{XOD} / \mathrm{XDH}$ activity in liver but not in serum. ${ }^{9}$ In line with these observations, we found that hepatic XOD/XDH activity played a key role in modulating uric acid level after Morin/ HES-DOCA-NPs treatment in the present study. In addition, Morin/HES-DOCA-NPs greatly improved uric acid excretion from the kidney, as evidenced by the increased $\mathrm{FE}_{\mathrm{UA}}$ value of NPs-treated rats. Our findings with the systemic delivery of Morin/HES-DOCA-NPs encouraged the continued development of HES-based polymers as a viable nanoplatform to prevent or treat additional diseases.

While the in vivo treatment study validates the therapeutic benefit of Morin/HES-DOCA-NPs, additional studies are necessary to further optimize this new formulation for clinical translation. Our future work will define the potential preferential size and surface properties of HES-based NP formulations during in vivo circulation. The therapeutic efficacy observed in this study was demonstrated in a short-term hyperuricemia rat model. Further studies need to be done in animals with longer term hyperuricemia.

\section{Conclusion}

In this study, we report the successful synthesis of a novel amphiphilic polymer based on HES, which could spontaneously self-assemble into stable NPs. The HES-based NPs selfassembled from optimized HES-DOCA conjugates enabled a high DL capacity of Morin. The systemic application of Morin/HES-DOCA-NPs remarkably extended the half-life of Morin and led to superior serum urate lowering effect in vivo. The success of this formulation provided a solid proof for the explorations of HES-based polymers as a viable strategy to extend the in vivo half-life of short-time bioactive drugs. Thus, we believe that the novel self-assembled HES NPs can be applied to additional therapeutic agents to improve the treatment of many other diseases.

\section{Acknowledgment}

We are thankful for the financial support provided by the National Natural Science Foundations of People's Republic of China (Grant Nos: 81703433 and 81703422).

\section{Disclosure}

The authors report no conflicts of interest in this work.

\section{References}

1. Jin M, Yang F, Yang I, et al. Uric acid, hyperuricemia and vascular diseases. Front Biosci (Landmark Ed). 2012;17(1):656-669.

2. Baker JF, Schumacher HR. Update on gout and hyperuricemia. Int $J$ Clin Pract. 2010;64(3):371-377.

3. Terkeltaub R. Update on gout: new therapeutic strategies and options Nat Rev Rheumatol. 2010;6(1):30-38. 
4. Kapoor R, Kakkar P. Protective role of morin, a flavonoid, against high glucose induced oxidative stress mediated apoptosis in primary rat hepatocytes. PLoS One. 2012;7(8):41663.

5. Subash S, Subramanian P. Morin a flavonoid exerts antioxidant potential in chronic hyperammonemic rats: a biochemical and histopathological study. Mol Cell Biochem. 2009;327(1-2):153-161.

6. Caselli A, Cirri P, Santi A, Paoli P. Morin: a promising natural drug. Curr Med Chem. 2016;23(8):774-791.

7. Yu Z, Fong WP, Cheng $\mathrm{CH}$. The dual actions of morin $\left(3,5,7,2^{\prime}, 4^{\prime}-\right.$ pentahydroxyflavone) as a hypouricemic agent: uricosuric effect and xanthine oxidase inhibitory activity. J Pharmacol Exp Ther. 2006; 316(1):169-175.

8. Sinha K, Ghosh J, Sil PC. Morin and its role in chronic diseases. Adv Exp Med Biol. 2016;928:453-471.

9. Zhang JJ, Shuai X, Li JB, Xiang NX, Gong T, Zhang ZR. Biodistribution, hypouricemic efficacy and therapeutic mechanism of morin phospholipid complex loaded self-nanoemulsifying drug delivery systems in an experimental hyperuricemic model in rats. $J$ Pharm Pharmacol. 2016;68(1):14-25.

10. Zhang J, Peng Q, Shi S, et al. Preparation, characterization, and in vivo evaluation of a self-nanoemulsifying drug delivery system (SNEDDS) loaded with morin-phospholipid complex. Int J Nanomedicine. 2011;6: 3405-3414.

11. Waddad AY, Abbad S, Yu F, et al. Formulation, characterization and pharmacokinetics of Morin hydrate niosomes prepared from various non-ionic surfactants. Int J Pharm. 2013;456(2):446-458.

12. Besheer A, Hause G, Kressler J, Mader K. Hydrophobically modified hydroxyethyl starch: synthesis, characterization, and aqueous self-assembly into nano-sized polymeric micelles and vesicles. Biomacromolecules. 2007;8(2):359-367.

13. Li G, Li Y, Tang Y, et al. Hydroxyethyl starch conjugates for improving the stability, pharmacokinetic behavior and antitumor activity of 10-hydroxy camptothecin. Int J Pharm. 2014;471(1-2):234-244.

14. Goszczyński TM, Filip-Psurska B, Kempińska K, Wietrzyk J, Boratyński J. Hydroxyethyl starch as an effective methotrexate carrier in anticancer therapy. Pharmacol Res Perspect. 2014;2(3):e00047.
15. Yang J, Gao C, Lü S, Zhang X, Yu C, Liu M. Physicochemical characterization of amphiphilic nanoparticles based on the novel starchdeoxycholic acid conjugates and self-aggregates. Carbohydr Polym. 2014;102:838-845.

16. Zhang J, Li J, Ju Y, Fu Y, Gong T, Zhang Z. Mechanism of enhanced oral absorption of morin by phospholipid complex based self-nanoemulsifying drug delivery system. Mol Pharm. 2015;12(2):504-513.

17. Besheer A, Vogel J, Glanz D, Kressler J, Groth T, Mader K. Characterization of PLGA nanospheres stabilized with amphiphilic polymers: hydrophobically modified hydroxyethyl starch vs pluronics. Mol Pharm. 2009;6(2):407-415.

18. Paleos CM, Sideratou Z, Tsiourvas D. Drug delivery systems based on hydroxyethyl starch. Bioconjugate Chemistry. 2017;28(6): 1611-1624.

19. Li J, Huo M, Wang J, et al. Redox-sensitive micelles self-assembled from amphiphilic hyaluronic acid-deoxycholic acid conjugates for targeted intracellular delivery of paclitaxel. Biomaterials. 2012;33(7): 2310-2320

20. Shapiro NI, Trzeciak S, Hollander JE, et al. The diagnostic accuracy of plasma neutrophil gelatinase-associated lipocalin in the prediction of acute kidney injury in emergency department patients with suspected sepsis. Ann Emerg Med. 2010;56(1):52-59.

21. Elhmidi Y, Bleiziffer S, Piazza N, et al. Incidence and predictors of acute kidney injury in patients undergoing transcatheter aortic valve implantation. Am Heart J. 2011;161(4):735-739.

22. Choi KY, Chung H, Min KH, et al. Self-assembled hyaluronic acid nanoparticles for active tumor targeting. Biomaterials. 2010;31(1): 106-114.

23. Baier G, Baumann D, Siebert JM, Musyanovych A, Mailander V, Landfester K. Suppressing unspecific cell uptake for targeted delivery using hydroxyethyl starch nanocapsules. Biomacromolecules. 2012; 13(9):2704-2715.

24. Lapsia V, Johnson RJ, Dass B, et al. Elevated uric acid increases the risk for acute kidney injury. Am J Med. 2012;125(3):e9-e17. 


\section{Supplementary material}

A

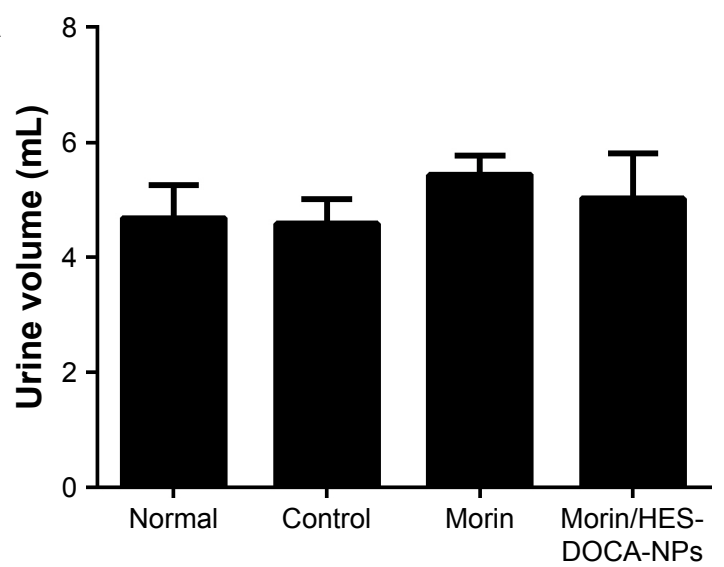

C

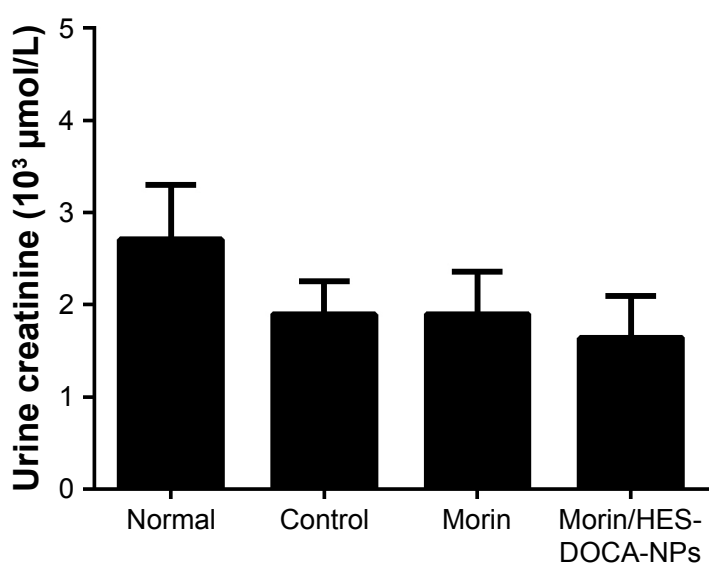

B

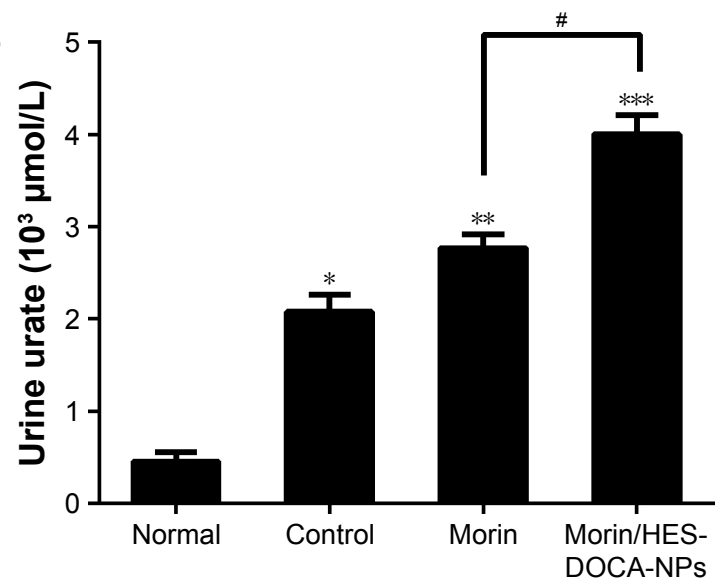

D

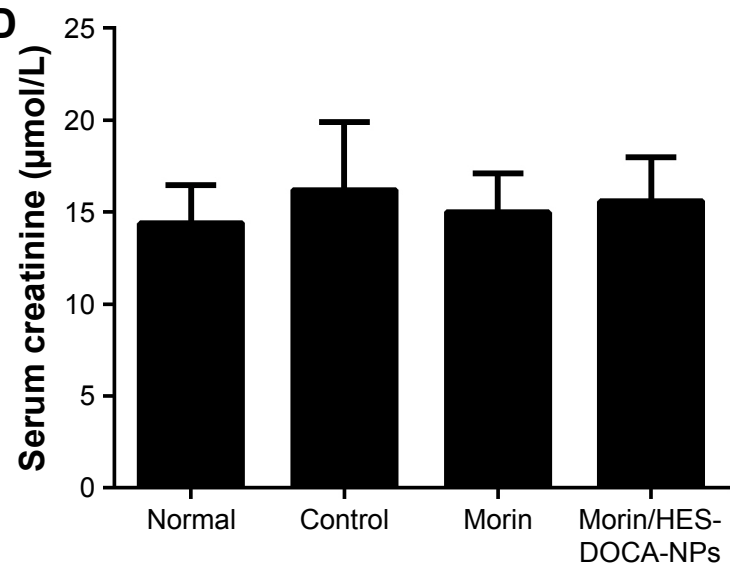

Figure SI Parameters including (A) urine volume, (B) urine urate level, (C) urine creatinine level and (D) serum creatinine level for calculating FE DOCA-NPs on hyperuricemic rats induced by potassium oxonate. Urine was collected for $5 \mathrm{~h}$. Blood sample was obtained by cardiopuncture at the end of the 5 -h period. Notes: Results are shown as mean \pm standard deviation $(\mathrm{n}=10)$. ${ }^{*} \mathrm{P}<0.05$, ${ }^{* *} \mathrm{P}<0.0 \mathrm{I}$, ${ }^{* * *} \mathrm{P}<0.00 \mathrm{I}$, compared with normal group. ${ }^{\#} \mathrm{P}<0.05$, compared with Morin group. Abbreviations: Morin/HES-DOCA-NPs, hydroxyethyl starch-deoxycholic acid nanoparticles-loaded Morin; $\mathrm{FE}_{\mathrm{UA}}$, fractional excretion of urate.

\section{Publish your work in this journal}

The International Journal of Nanomedicine is an international, peerreviewed journal focusing on the application of nanotechnology in diagnostics, therapeutics, and drug delivery systems throughout the biomedical field. This journal is indexed on PubMed Central, MedLine, CAS, SciSearch ${ }^{\circledR}$, Current Contents ${ }^{\circledR} /$ Clinical Medicine,
Journal Citation Reports/Science Edition, EMBase, Scopus and the Elsevier Bibliographic databases. The manuscript management system is completely online and includes a very quick and fair peer-review system, which is all easy to use. Visit http://www.dovepress.com/ testimonials.php to read real quotes from published authors. 